\title{
Ocular neovascularization in eyes with a central retinal artery occlusion or a branch retinal artery occlusion
}

This article was published in the following Dove Press journal:

Clinical Ophthalmology

5 June 2015

Number of times this article has been viewed

\author{
John O Mason III', \\ Shyam A Patel' \\ Richard M Feist ${ }^{1,2}$ \\ Michael A Albert Jr ${ }^{1,2}$ \\ Carrie Huisingh' \\ Gerald McGwin Jr', \\ Martin L Thomley ${ }^{1,2}$ \\ 'Department of Ophthalmology, \\ University of Alabama School of \\ Medicine, Birmingham, AL, USA; \\ ${ }^{2}$ Retina Consultants of Alabama, \\ Callahan Eye Foundation Hospital, \\ Birmingham, AL, USA; ${ }^{3}$ Department of \\ Epidemiology, School of Public Health, \\ University of Alabama at Birmingham, \\ Birmingham, AL, USA
}

Correspondence: John O Mason III Retina Consultants of Alabama, Callahan Eye Foundation Hospital, 700 South 18th Street, Suite 707, Birmingham, AL 35233, USA

Tel +l 2059180047

Email masonallmason@yahoo.com
Purpose: To investigate the ocular neovascularization (ONV) rate in eyes with a branch retinal artery occlusion (BRAO) or a central retinal artery occlusion (CRAO), and to study factors that may influence the ONV rate secondary to CRAO.

Methods: This was a retrospective case series of consecutive patients (286 total eyes: 83 CRAOs and 203 BRAOs) who were diagnosed with a retinal artery occlusion from 1998 to 2013 at the Retina Consultants of Alabama and University of Alabama at Birmingham, Birmingham, AL, USA. Generalized estimating equations were used to evaluate the association between hypothesized risk factors and ONV development.

Results: Twelve (14.5\%) of the 83 eyes with a CRAO developed ONV. Eleven of 12 eyes $(91.7 \%)$ had iris neovascularization, ten of 12 eyes $(83.3 \%)$ had neovascular glaucoma, and two of 12 eyes $(16.7 \%)$ had neovascularization of the optic disc. The average time for ONV development secondary to CRAO was 30.7 days, ranging from the date of presentation to 137 days. Only two $(<1.0 \%)$ of the 203 eyes with a BRAO developed iris neovascularization. Diabetes mellitus type 2 was a risk factor for ONV development following a CRAO with an adjusted odds ratio of 5.2 (95\% confidence interval: $1.4-19.8)(P=0.02)$.

Conclusion: $\mathrm{ONV}$ is an important complication of $\mathrm{CRAO}$ and is a less-frequent complication of BRAO. Patients with a CRAO, especially those with diabetes mellitus type 2 , should be closely monitored for the first 6 months for ONV.

Keywords: neovascularization, neovascular glaucoma, retinal artery occlusion, central retinal artery occlusion, branch retinal artery occlusion, diabetes mellitus

\section{Introduction}

Central retinal artery occlusion (CRAO) is an ophthalmic emergency in which the central retinal artery is occluded, resulting in a retinal infarct and acute vision loss. Its incidence is estimated to be one in $100,000 .{ }^{1}$ Analogous to a cerebrovascular accident (CVA), CRAOs share the same risk factors and etiologies of a CVA, with the most common etiology being an embolus, usually arising from the carotid artery. ${ }^{2}$ Even though visual improvement can occur in patients with CRAOs, Hayreh and Zimmerman found that $71.5 \%$ of CRAO eyes had a final visual acuity of 20/400 or worse. ${ }^{3}$ Therefore, frequent follow-up visits to monitor visual acuity and to assess risk factors for future CVAs are needed in these patients.

CRAOs are associated with complications like ocular neovascularization (ONV). The occurrence rate of ONV in eyes with a CRAO varies from $3.0 \%$ to $18.8 \%$ in studies conducted during the past 35 years. ${ }^{2,4-10}$ Furthermore, the detection of ONV following CRAO has ranged from as early as the day of presentation to 2 years after the CRAO diagnosis. ${ }^{4,8}$ With frequent visits, many cases of ONV can be managed early with 
treatments such as panretinal photocoagulation and off-label intravitreal bevacizumab. ${ }^{9,11,12}$ Rudkin et al concluded that since ONV can occur early, regular follow-up appointments should be required, especially within the first 4 months. ${ }^{5}$

The incidence of ONV as a result of branch retinal artery occlusion (BRAO) has not been well documented. Brown and Reber theorized that there was a narrow window for an ischemic retina to produce angiogenic factors because of possible spontaneous reestablishment of retinal blood flow and more rapid cellular damage rather than chronic prolonged ischemic damage as seen in other retinal vascular conditions. ${ }^{13}$ In addition, ONV is likely related to the degree of occlusion and area of ischemia, which is smaller in a BRAO when compared with a CRAO. Thus, it was presumed that the incidence of BRAO-induced neovascularization was rare. In fact, Hayreh and Podhajsky reported no incidence of ONV in 44 eyes with BRAO. ${ }^{4}$

In this study, our purpose was to investigate ONV development in eyes with a BRAO or a CRAO and associated possible systemic risk factors.

\section{Methods}

The medical records of consecutive patients with a diagnosis of CRAOs and BRAOs made at the Retina Consultants of Alabama and the University of Alabama at Birmingham (UAB), Birmingham, AL, USA between 1998 and 2013 were reviewed. The Institutional Review Board of UAB approved this study, and this study was Health Insurance Portability and Accountability Act (HIPAA) compliant. Our study included 83 eyes with a CRAO and 203 eyes with a BRAO. All eyes included had a documented clinical diagnosis of CRAO or BRAO made by one of the practicing retinologists. Charts with incomplete information, questionable diagnosis, presence of ONV before retinal artery occlusion, multiple BRAOs in the same eye, or other likely etiologies of ONV were omitted from this study. Other likely etiologies omitted included vein occlusions, proliferative diabetic retinopathy, ocular ischemia syndrome, radiation retinopathy, and uveitis.

All charts were reviewed for complications of ONV, neovascularization of the iris (NVI), neovascular glaucoma (NVG), neovascularization of the optic disc (NVD), the timing of ONV development, the peak intraocular pressure (IOP) in patients with NVG, and the treatment used for $\mathrm{NVG}$, including panretinal photocoagulation and treatment for elevated IOP. In this study, NVG was defined as the presence of NVI with an IOP greater than $22 \mathrm{mmHg}$ without prior history of glaucoma or ocular hypertension. ${ }^{14}$ Patient charts with a CRAO diagnosis were further analyzed for a diagnosis of temporal arteritis (blood evaluation of erythrocyte sedimentation rate, C-reactive protein), visible emboli on initial dilated fundus exam, ipsilateral internal carotid artery (ICA) Doppler ultrasound results (or cerebral angiography), and echocardiogram results.

Additional variables, such as age, sex, other ocular conditions, and other comorbidities, were also recorded for eyes with a CRAO. These comorbidities included hypertension, diabetes mellitus type 2 (DM2), hyperlipidemia (HLD), history of CVA, and smoking history. These diagnoses were made based on medical history from a primary care physician or the current use of a medication commonly used for hypertension, DM2, and HLD, respectively. Variables and risk factors for ONV following a BRAO were not analyzed due to the low prevalence of ONV secondary to BRAO in this study population.

Demographic and clinical characteristics were compared between CRAO eyes with and without ONV. Logistic regression using generalized estimating equations were used to calculate crude and adjusted odds ratios (ORs) and corresponding 95\% confidence intervals (CIs); generalized estimating equations are necessary to account for withinsubject correlation among fellow eyes from the same individual. The percent of BRAO eyes that developed ONV was also reported. A $P$-value of less than 0.05 was considered statistically significant. SAS 9.3 was used for all statistical analysis.

\section{Results}

There were 83 eyes (from $n=81$ people) with a CRAO diagnosis and 203 (from $n=203$ people) with a BRAO diagnosis. The average age of our CRAO patients was 69.2 years (ranging from 19 to 95 years), and 40 (48\%) eyes occurred in males (Table 1). The average age of the patients with ONV in eyes with a CRAO was 66.5 (standard deviation 9.3) years, as compared with 69.7 (standard deviation 15.3) years for patients with no ONV. The CRAO was diagnosed in the right eye in $53 \%$ of all patients.

Twelve (14.5\%) of these 83 eyes with CRAO developed ONV (Table 2). The mean time from diagnosis of CRAO to development of ONV was 30.7 days (ranging from the date of presentation to 137 months after CRAO diagnosis). Eleven $(91.7 \%)$ of 12 eyes with ONV had NVI, ten $(83.3 \%)$ eyes had NVG, and two (16.7\%) eyes had NVD. Ten of the 12 patients with ONV had multiple vascular comorbidities.

As summarized in Table 3, those with ONV were more likely to be male compared to those without ONV (OR =3.9, 95\% CI: 1.0-15.6); however, this association was not 
Table I Baseline characteristics of eyes with a CRAO

\begin{tabular}{|c|c|c|c|}
\hline Characteristic & Total $(n=83)$ & With ONV $(n=12)$ & Without ONV $(n=7 I)$ \\
\hline \multicolumn{4}{|l|}{ Sex, n (\%) } \\
\hline Male & $40(48 \%)$ & $9(75 \%)$ & 31 (44\%) \\
\hline Female & 43 (52\%) & $3(25 \%)$ & $40(56 \%)$ \\
\hline \multicolumn{4}{|l|}{ Age, years } \\
\hline Mean (SD) & $69.2(14.6)$ & $66.5(9.3)$ & $69.7(15.3)$ \\
\hline Minimum-maximum & $16.6-95.7$ & $46.7-76.9$ & 16.6-95.7 \\
\hline \multicolumn{4}{|l|}{ Eye with CRAO } \\
\hline OD & 44 (53\%) & $4(33 \%)$ & $40(56 \%)$ \\
\hline OS & 39 (47\%) & $8(67 \%)$ & 31 (44\%) \\
\hline \multicolumn{4}{|l|}{ Systemic conditions, n (\%) } \\
\hline Hypertension & $63(76 \%)$ & $10(83 \%)$ & $53(75 \%)$ \\
\hline Hyperlipidemia & 40 (48\%) & 7 (58\%) & $33(46 \%)$ \\
\hline CVA & $28(34 \%)$ & $5(42 \%)$ & $23(32 \%)$ \\
\hline Diabetes mellitus type 2 & 27 (33\%) & $8(67 \%)$ & $19(27 \%)$ \\
\hline History of smoking & $30(37 \%)$ & $5(42 \%)$ & $25(36 \%)$ \\
\hline Temporal arteritis & $2(2 \%)$ & I (8\%) & I (I\%) \\
\hline Visible emboli on DFE & $10(12 \%)$ & $0(0 \%)$ & $10(14 \%)$ \\
\hline Severe ipsilateral internal carotid artery stenosis $(>60 \%)$ & $8(10 \%)$ & $2(17 \%)$ & $6(8 \%)$ \\
\hline Thrombus or vegetation of valve on $\mathrm{ECHO}$ & $4(5 \%)$ & $0(0 \%)$ & $4(6 \%)$ \\
\hline
\end{tabular}

Abbreviations: CRAO, central retinal artery occlusion; CVA, cerebrovascular accident; DFE, dilated fundus exam; ECHO, echocardiogram; OD, right eye; OS, left eye; ONV, ocular neovascularization; SD, standard deviation.

statistically significant. With respect to comorbid conditions, those with ONV were more likely to have DM2 compared to those without ONV (OR $=5.5,95 \%$ CI: $1.5-20.3)$. ONV was not significantly associated with the other conditions examined. After adjusting for the potentially confounding effects of sex, the association between ONV and DM2 persisted (adjusted OR $=5.2,95 \%$ CI: 1.4-19.8, $P=0.02$ ).

Two (1.0\%) of the 203 eyes with a BRAO developed ONV in the form of NVI (Table 4). The average time for ONV development was 4.5 months in these two eyes. A 33-yearold, white female patient developed NVI 3 months after a quadrantic inferotemporal BRAO, and an 82-year-old, white male developed NVI 6 months after a quadrantic superotemporal BRAO. Both patients had thorough systemic diagnostic work-ups, which were negative. Fortunately, laser photocoagulation in the distribution of the quadrantic BRAO ischemic retina was effective in treating NVI in both patients.

\section{Discussion CRAOs and ONV}

We found that $14.5 \%$ of eyes with a CRAO had ONV. All patients were followed for at least 1 year. This is consistent with prior studies, which have reported between $3.0 \%$ and

Table 2 Eyes with ocular neovascularization following a CRAO

\begin{tabular}{|c|c|c|c|c|c|c|c|}
\hline Eye & NVD & NVI & NVG & $\begin{array}{l}\text { Peak IOP }(\mathrm{mmHg}) \\
\text { on medication }\end{array}$ & $\begin{array}{l}\text { Time from onset } \\
\text { of CRAO diagnosis }\end{array}$ & Other comorbidities & Management of NVG \\
\hline I & No & Yes & Yes & 26 & Same day & HTN, CVA, SH & PRP, IVR, tube shunt \\
\hline 2 & Yes & Yes & Yes & 48 & 48 days & HTN, DM2, HLD, SH & PRP, IVB \\
\hline 3 & No & Yes & Yes & 43 & 62 days & HTN, DM2, HLD & IVB, tube shunt \\
\hline 4 & No & Yes & Yes & 60 & 32 days & $\mathrm{DM} 2$ & Retrobulbar thorazine \\
\hline 5 & No & Yes & Yes & 32 & Same day & HTN, DM2, HLD, CVA & IVB, tube shunt \\
\hline 6 & No & Yes & Yes & 71 & Same day & $\mathrm{HTN}$ & IVB \\
\hline 7 & No & Yes & No & N/A & Same day & HTN, DM2, HLD, CVA & N/A \\
\hline 8 & No & Yes & Yes & 38 & Same day & HTN, DM2, HLD & PRP, IVB \\
\hline 9 & No & Yes & Yes & 23 & 43 days & HTN, HLD, CVA & PRP, IVB \\
\hline 10 & Yes & No & No & $\mathrm{N} / \mathrm{A}$ & 167 days & DM2, HLD, SH & N/A \\
\hline II & No & Yes & Yes & 46 & Same day & HTN, DM2, SH & No therapy recorded \\
\hline 12 & No & Yes & Yes & 28 & 44 days & HTN, CVA, SH, TA & PRP \\
\hline
\end{tabular}

Abbreviations: CRAO, central retinal artery occlusion; CVA, cerebrovascular accident; DM2, diabetes mellitus type 2; HLD, hyperlipidemia; HTN, hypertension; IOP, intraocular pressure; IVB, intravitreal bevacizumab; IVR, intravitreal ranibizumab; N/A, not applicable; NVD, neovascularization of the optic disc; NVG, neovascular glaucoma; $\mathrm{NVI}$, neovascularization of the iris; PRP, panretinal photocoagulation; SH, smoking history; TA, temporal arteritis. 
Table 3 Association between risk factors and development of ocular neovascularization in eyes with a central retinal artery occlusion

\begin{tabular}{llll}
\hline & Crude OR & $\mathbf{9 5 \%} \mathbf{C l}$ & $\mathbf{P}$-value \\
\hline Male (reference female) & 3.9 & $1.0-15.6$ & 0.06 \\
Hypertension & 1.7 & $0.3-8.6$ & 0.52 \\
Hyperlipidemia & 1.6 & $0.5-5.6$ & 0.45 \\
CVA & 1.5 & $0.4-5.2$ & 0.53 \\
Diabetes mellitus type 2 & 5.5 & $1.5-20.3$ & 0.01 \\
History of smoking & 1.3 & $0.4-4.5$ & 0.69 \\
Temporal arteritis & 6.4 & $0.4-109.4$ & 0.20 \\
Visible emboli on DFE & - & - & - \\
Severe ipsilateral internal carotid artery stenosis $(>60 \%)$ & 2.2 & $0.4-12.3$ & 0.38 \\
Thrombus or vegetation of valve on ECHO & - & - & - \\
\hline
\end{tabular}

Note: Reference is those without the condition, unless otherwise specified.

Abbreviations: $\mathrm{Cl}$, confidence interval; CVA, cerebrovascular accident; DFE, dilated fundus exam; ECHO, echocardiogram; OR, odds ratio.

$18.8 \%$ of CRAO eyes having ONV., ${ }^{2,-10}$ From these prior eight studies, Hayreh et al's study in 2009 reported that $3.0 \%$ of CRAO eyes had ONV. ${ }^{2}$ The next lowest ONV rate was $16.6 \%{ }^{7}$ Furthermore, Hayreh et al stated that there was no cause and effect relationship between CRAO and NVG. ${ }^{2}$ Their reasoning was that ocular ischemia can be present in eyes even without significant findings on ICA Doppler ultrasound studies and that other arteries, such as the ophthalmic artery, can be occluded without any significant occlusion of the ipsilateral ICA. ${ }^{15,16}$ Therefore, they inferred that many of the patients in other studies likely had ocular ischemia, despite having normal ICA studies, and that the underlying ocular ischemia caused the ONV.

Sagong et al made a valid point in their study of three eyes with NVG following a CRAO. ${ }^{9}$ They stated that the timing of all three patients developing NVG after the CRAO, in addition to the patients having no signs of ONV prior to the CRAO, strongly suggests an association between NVG and CRAO. In addition, their three patients had no delay in filling on fluorescein angiogram. ${ }^{9}$ More recently, in 2010, Rudkin et al stated that they demonstrated a clear correlation and a "causal relationship" between CRAO and NVI based on the timing of the two entities. Also, the majority of their cases had no evidence of ocular ischemia and no significant carotid artery stenosis. ${ }^{5}$

Our study findings agree with Sagong et al and Rudkin et al's studies and conclusions about an existing association between CRAO and ONV. Excluding other etiologies of neovascularization, three out of our five patients with ONV and available ICA study results had no significant stenosis. ${ }^{5,9}$

However, in the case of ocular ischemia syndrome, Hayreh found the rate of ipsilateral ICA was severe $(>80 \%$ occlusion) in $74 \%$ of the patients. ${ }^{16}$ Therefore, this likely represents that not having at least significant ( $>60 \%$ occlusion) stenosis in the ipsilateral ICA in patients with ocular ischemic syndrome is definitely in the minority. Further supporting this, Brown et al's study stated that most patients that have ocular ischemia have either bilateral common carotid artery obstruction or severe bilateral obstruction of the ICAs. ${ }^{17}$

Our results agree with Hayreh and Podhajsky's study, which found that ONV may develop by the time of patient presentation. ${ }^{4}$ Six of our 12 patients with ONV following CRAO had neovascularization at the time of presentation, and we feel that examination for ONV should begin in patients with CRAO on the day of presentation. Our time range of development for ONV in eyes with a CRAO was from the date of presentation to about 5 months after the CRAO diagnosis. Five of the previous eight studies had a time range for the development of ONV that fell within our range..$^{5-7,9,10}$ Therefore, we suggest that CRAO patients should be monitored at a minimum for the first 5 months for signs of ONV.

This is the first study to find that DM2 is a risk factor for $\mathrm{ONV}$, as it was highly associated with ONV development

Table 4 Patients with ONV following a BRAO

\begin{tabular}{|c|c|c|c|c|c|c|c|c|c|}
\hline Patient & $\begin{array}{l}\text { Age } \\
\text { (years) }\end{array}$ & Sex & Race & $\begin{array}{l}\text { Location } \\
\text { of BRAO }\end{array}$ & $\begin{array}{l}\text { Risk Factors } \\
\text { for BRAO }\end{array}$ & $\begin{array}{l}\text { Timing } \\
\text { of ONV }\end{array}$ & NVI & NVG & Treatment \\
\hline I & 82 & Male & White & Superotemporal & None & 6 months & Yes & No & Photocoagulation \\
\hline 2 & 33 & Female & White & Inferotemporal & None & 3 months & Yes & No & Photocoagulation \\
\hline
\end{tabular}

Abbreviations: BRAO, branch retinal artery occlusion; NVG, neovascular glaucoma; NVI, neovascularization of the iris; ONV, ocular neovascularization. 
in eyes with a CRAO (Tables 1 and 3). DM2 had a high OR of 5.2 in patients with ONV. DM2 is considered a vascular disease, and even though none of our patients had proliferative diabetic retinopathy, some patients may have had enough undetected microvascular occlusion to cause chronic hypoxia. In fact, Blann et al noted that vascular endothelial growth factor, the main signal involved in development of ONV, was elevated in patients with both DM2 and atherosclerosis. ${ }^{18}$ Our only mode of diagnosis of atherosclerosis in this study was ICA Doppler ultrasound studies. Only two patients in our study with DM2 had significant $(>60 \%)$ or greater stenosis of the ipsilateral ICA, but ICA study results were only found on ten patients with DM2. Fifteen of the 28 eyes with DM2 had HLD predisposing them to atherosclerosis. Furthermore, 25 (89\%) of these 28 eyes had multiple vascular comorbidities. Therefore, it is very likely that many of our patients with DM2 had underlying atherosclerosis, and we believe patients with CRAO and DM2 need to be monitored very closely for ONV.

\section{BRAOs and ONV}

BRAOs are usually attributed to emboli from the extracranial carotid arteries or heart, though non-embolic causes of arterial occlusion include various vasculitides, coagulopathies, and inflammatory processes. BRAO is thought to represent $38 \%$ of all arterial vaso-occlusions in the eye. ${ }^{19}$ Visual outcomes may depend on presenting visual acuity, which likely represents the initial severity of retinal ischemia and the presence of a perfused cilioretinal artery. Mason et al showed that only two (14\%) eyes with a presenting visual acuity worse than $20 / 100$ improved to $20 / 40$ or better. They also found that $25(89 \%)$ eyes with a visual acuity of 20/40 or better remained with good vision. ${ }^{20}$ One previous study has reported that $80 \%$ of individuals with BRAO maintain visual acuity of $20 / 40$ or better. ${ }^{21}$

ONV is thought to be rare after a BRAO compared with CRAO. An and Kwon explain that this could possibly be due to an insufficient quantity of angiogenic factors being produced. ${ }^{22}$ Hayreh and Podhajsky found the incidence of NVI to be $0 \%$ after following 44 eyes with non-recurrent BRAO. ${ }^{4}$ There have been only two published cases of an isolated BRAO-induced NVI or NVG. Shah et al documented one case where a 70 -year-old male, with $30 \%$ stenosis of the ipsilateral common carotid artery, developed NVI (without neovascularization of the angle) and NVD 1 month after BRAO. ${ }^{23}$ Likewise, Wasik and Dolan described a case of a 68-year-old male with only mild carotid insufficiency who developed NVG 4 months after a BRAO in his right eye. ${ }^{24}$
In our study, we found two of 203 eyes developed ONV and NVI within 6 months of BRAO diagnosis.

Compared with isolated BRAO-induced NVI and NVG, there are a greater number of recurrent BRAO-induced NVI and NVG cases described in the literature. Yamamoto et al described a 72-year-old male with a cardiac thrombus who developed NVG only 3 days after the second BRAO, which was 5 weeks after the first BRAO. ${ }^{25}$ Bresnick and Gay studied a patient who developed bilateral NVI 3 months after occlusions of multiple temporal branch retinal arteries, which were later attributed to a cardiac mural thrombus. ${ }^{26}$ Similarly, Capone and Meredith mentioned a case of a healthy 65-yearold male who experienced NVI, NVG, and disc neovascularization after multiple, idiopathic BRAOs. ${ }^{27}$

The current results are consistent with the conclusion that ONV after BRAO is rare. The ONV was presumed to be secondary to the BRAO as neither of our two patients had carotid occlusion, signs of ocular ischemia syndrome, diabetes mellitus, vasculitis, or any abnormality on systemic workup. Following laser photocoagulation to the area of BRAO, the NVI regressed entirely in both eyes, and neither eye developed NVG. Similarly, Shah et al used photocoagulation for their patient, which led to regression of both NVD and NVI. ${ }^{23}$ In a case of NVG induced by BRAO, Yamamoto et al described photocoagulation, which eventually led to neovascular regression and normal IOP. ${ }^{25}$ Capone and Meredith also support the use of photocoagulation as it was successfully used in their case of recurrent BRAO-induced NVI and NVG. ${ }^{27}$

We recognize the limitations of this study, including its retrospective nature, lack of control group, and that the follow-up period varied considerably between patients. In addition, there was no control group for comparisons. Due to its retrospective nature, a few patient charts were excluded from our study because of incomplete data, and certain data, such as ICA Doppler ultrasound, were not available for all patients. Nevertheless, we feel that our study has a very large number of patients with long follow-up (at least 1 year) and sufficient data to make valid conclusions.

\section{Conclusion}

ONV is an important and common complication of eyes with a CRAO. This is especially true for patients with DM2, which was found to be highly associated with ONV in eyes with a CRAO. Furthermore, BRAO-induced ONVs in the form of NVI are much less common than in CRAOs. Due to the threat of further visual loss from NVG, we recommend continued careful observation, at least in the early follow-up period (0 to 6 months), for the development of ONV in eyes 
with retinal artery occlusions, particularly in DM2 patients with a CRAO.

\section{Acknowledgments}

Financial support was received from Research to Prevent Blindness, New York, NY, USA. The sponsor had no role in the design or conduct of this research. The work was conducted at the University of Alabama School of Medicine, Birmingham, AL, USA. An abstract of much of the data presented in this paper was presented at the American Academy of Ophthalmology Annual Meeting (Chicago, IL, USA in October 2014).

\section{Disclosure}

The authors report no conflicts of interest in this work.

\section{References}

1. Rumelt S, Dorenboim Y, Rehany U. Aggressive systematic treatment for central retinal artery occlusion. Am J Ophthalmol. 1999;128:733-738.

2. Hayreh SS, Podhajsky PA, Zimmerman MB. Retinal artery occlusion: associated systemic and ophthalmic abnormalities. Ophthalmology. 2009;116(10):1928-1936.

3. Hayreh SS, Zimmerman MB. Central retinal artery occlusion: visual outcome. Am J Ophthalmol. 2005;140(3):376-391.

4. Hayreh SS, Podhajsky P. Ocular neovascularization with retinal vascular occlusion. II. Occurrence in central and branch retinal artery occlusion. Arch Ophthalmol. 1982;100(10):1585-1596.

5. Rudkin AK, Lee AW, Chen CS. Ocular neovascularization following central retinal artery occlusion: prevalence and timing of onset. Eur J Ophthalmol. 2010;20(6):1042-1046.

6. Duker JS, Brown GC. Iris neovascularization associated with obstruction of the central retinal artery. Ophthalmology. 1988;95(9):1244-1250.

7. Duker JS, Sivalingam A, Brown GC, Reber R. A prospective study of acute central retinal artery obstruction. The incidence of secondary ocular neovascularization. Arch Ophthalmol. 1991;109:339-342.

8. Schäfer S, Lang GE. [Iris neovascularization as a complication of central artery occlusion]. Klin Monbl Augenheilkd. 2005;222(4):343-345. German.

9. Sagong M, Kim J, Chang W. Intravitreal bevacizumab for the treatment of neovascular glaucoma associated with central retinal artery occlusion. Korean J Ophthalmol. 2009;23(3):215-218.

10. Kattah JC, Wang DZ, Reddy C. Intravenous recombinant tissue-type plasminogen activator thrombolysis in treatment of central retinal artery occlusion. Arch Ophthalmol. 2002;120:1234-1236.
11. Duker JS, Brown GC. The efficacy of panretinal photocoagulation for neovascularization of the iris after central retinal artery obstruction. Ophthalmology. 1989;96(1):92-95.

12. Vatavuk Z, Bencic G, Mandic Z. Intravitreal bevacizumab for neovascular glaucoma following central retinal artery occlusion. Eur J Ophthalmol. 2007;17(2):269-271.

13. Brown GC, Reber R. An unusual presentation of branch retinal artery obstruction in association with ocular neovascularization. Can J Ophthalmol. 1986;21(3):103-106.

14. Zhang HT, Yang YX, Xu YY, Yang RM, Wang BJ, Hu JX. Intravitreal bevacizumab and Ahmed glaucoma valve implantation in patients with neovascular glaucoma. Int J Ophthalmol. 2014;7(5):837-842.

15. Mizener JB, Podhajsky P, Hayreh SS. Ocular ischemic syndrome. Ophthalmology. 1997;104:859-864.

16. Hayreh SS, Dass R. The ophthalmic artery: I. Origin and intra-cranial and intra-canalicular course. Br J Ophthalmol. 1962;46:65-98.

17. Brown GC, Magargal LE, Simeone FA, Goldberg RE, Federman JL, Benson WE. Arterial obstruction and ocular neovascularization. Ophthalmology. 1982;89(2):139-146.

18. Blann AD, Belgore FM, McCollum CN, Silverman S, Lip PL, Lip GY. Vascular endothelial growth factor and its receptor, Flt-1, in the plasma of patients with coronary or peripheral atherosclerosis, or type II diabetes. Clin Sci (Lond). 2002;102(2):187-194.

19. Brown GC, Shields JA. Cilioretinal arteries and retinal arterial occlusions. Arch Ophthalmol. 1979;97(1):84-92.

20. Mason JO 3rd, Shah AA, Vail RS, Nixon PA, Ready EL, Kimble JA. Branch retinal artery occlusion: visual prognosis. Am J Ophthalmol. 2008;146:455-457.

21. Brown GC, Magargal LE, Shields JA, Goldberg RE, Walsh PN. Retinal arterial obstruction in children and young adults. Ophthalmology. 1981;88(1):18-25.

22. An TS, Kwon SI. Neovascular glaucoma due to branch retinal vein occlusion combined with branch retinal artery occlusion. Korean $J$ Ophthalmol. 2013;27(1):64-67.

23. Shah GK, Sharma S, Brown GC. Iris neovascularization following branch retinal artery occlusion. Can J Ophthalmol. 1998;33(7):389-390.

24. Wasik AJ, Dolan BJ. Neovascular glaucoma following an isolated branch retinal artery occlusion. Clinical and Refractive Optometry. 2009; 20(1):18-22.

25. Yamamoto K, Tsujikawa A, Hangai M, Fujihara M, Iwawaki T, Kurimoto Y. Neovascular glaucoma after branch retinal artery occlusion. Jpn J Ophthalmol. 2005;49:388-390.

26. Bresnick GH, Gay AJ. Rubeosis iridis with branch retinal arteriolar occlusions. Arch Ophthalmol. 1967;77(2):176-180.

27. Capone A Jr, Meredith TA. Profound central visual loss and ocular neovascularization in idiopathic recurrent branch retinal arterial occlusion. Retina. 1990;10(4):265-268.

\section{Clinical Ophthalmology}

\section{Publish your work in this journal}

Clinical Ophthalmology is an international, peer-reviewed journal covering all subspecialties within ophthalmology. Key topics include: Optometry; Visual science; Pharmacology and drug therapy in eye diseases; Basic Sciences; Primary and Secondary eye care; Patient Safety and Quality of Care Improvements. This journal is indexed on Submit your manuscript here: http://www.dovepress.com/clinical-ophthalmology-journal

\section{Dovepress}

PubMed Central and CAS, and is the official journal of The Society of Clinical Ophthalmology (SCO). The manuscript management system is completely online and includes a very quick and fair peer-review system, which is all easy to use. Visit http://www.dovepress.com/ testimonials.php to read real quotes from published authors. 